\title{
Behavioral disruption versus signal-generated memory retrieval as determinants of the signal-generated partial reinforcement extinction effect
}

\author{
STEVEN J. HAGGBLOOM and VICKIE R. BREWER \\ Arkansas State University, State University, Arkansas
}

\begin{abstract}
In both experiments reported here, two groups of rats, Groups Signal-N and Disrupt, received runway discrimination training in Phase 1 to establish a stimulus as a signal for nonreinforcement (N). Group CRF received reinforcement throughout Phase 1. All subjects received consistent reinforcement throughout Phase 2. On 50\% of Phase 2 trials, Group Signal-N experienced the signal for nonreinforcement, and Group Disrupt experienced a novel stimulus. Both stimuli disrupted responding. In Experiment 1, the signal for $\mathrm{N}$ was a tactile cue, and the disruptive stimulus was a pretrial feeding. This arrangement was reversed in Experiment 2. In both experiments, Group Signal-N showed increased resistance to extinction, relative to Group CRF-a difference that defines the signal-generated partial reinforcement extinction effect (PREE) (Haggbloom, 1988). Resistance to extinction was not increased in Group Disrupt. The results indicate that the signal-generated PREE is not due simply to counterconditioning to behaviorally disruptive stimuli.
\end{abstract}

The signal-generated partial reinforcement extinction effect (PREE) (Haggbloom, 1988), like the conventional PREE, refers to increased resistance to extinction relative to consistent reinforcement (CRF). Unlike the conventional PREE, however, the signal-generated PREE occurs without the use of partial reinforcement (PRF) in the critical phase of training. Instead, a stimulus established as a signal for nonreinforcement $(\mathrm{N})$ in an initial phase of discrimination training is presented on selected trials in a subsequent phase of CRF training. These procedural differences notwithstanding, Haggbloom (1988) has suggested that the two PREEs are mediated by a common mechanism, namely, the acquired capacity of the memory of nonreinforcement $\left(S^{N}\right)$ to signal reinforcement $(R)$ and thus elicit fast running in extinction.

Two sources of goal-event memories, such as $\mathbf{S}^{\mathbf{N}}$, can be identified. We will refer to the memory of a goal event stored on one trial and retrieved on the very next trial as event-generated memory. The representation of a temporally remote goal event, which can be activated by presentation of a signal with which the memory is associated, will be called signal-generated memory.

It is well-established that extinction behavior is extensively controlled by the capacity of event-generated $S^{\mathbf{N}}$ to elicit running, and Capaldi (1966) has described in detail how that capacity is acquired during conventional PRF

These experiments were supported by a Faculty Research Committee Grant to the first author, from Arkansas State University. Requests for reprints should be addressed to Steven J. Haggbloom, Department of Psychology, Arkansas State University, P.O. Box 2127, State University, AR 72467. training. In what follows, we will describe procedures used to obtain the signal-generated PREE, its explanation in terms of signal-generated memory processes, and the rationale for the two experiments reported here, which tested an alternative interpretation derived from Amsel's (1972) generalized frustration theory.

Haggbloom (1988) trained rats on a feature-negative runway discrimination problem in Phase 1 , in order to establish either a pretrial feeding, or wire mesh laid on the runway floor, as a signal for nonreinforcement. The subjects in Group Signal-N then experienced the signal for nonreinforcement on selected $R$ trials during Phase 2 CRF training. It was hypothesized that $S^{N}$ would be stored in association with the signal during Phase 1 , and that presentation of the signal in Phase 2 would provoke $S^{\mathbf{N}}$ to an active state, resulting in learning about signalgenerated $S^{N}$ that otherwise would not occur. These procedures substantially increased resistance to extinction in the Signal-N subjects as opposed to CRF and nonsignaled control groups. Indeed, the Signal-N subjects were as resistant to extinction as subjects given actual PRF training in Phase 2. These results were interpreted as evidence for the acquisition of behavioral control by signalgenerated $\mathbf{S}^{\mathrm{N}}$, and-because extinction is regulated by event-generated $\mathrm{S}^{\mathrm{N}}$-for a high degree of similarity and hence transfer between signal-generated and eventgenerated $S^{N}$.

In the experiments reported by Haggbloom (1988), the presentation of the signal for $\mathbf{N}$ in Phase 2 disrupted instrumental responding, in that running speeds were very much slower on signaled than on unsignaled trials-a difference that abated over the course of Phase 2 train- 
ing. Amsel (1972) suggested that resistance to extinction is increased by counterconditioning of goal approach responses to behaviorally disruptive stimuli. The behavioral disruption initially produced by frustrative reactions to nonreinforcement during PRF training is a case in point, but counterconditioning to other disruptive stimulus events is predicted to produce a similar increase in resistance to extinction. An important assumption of Amsel's model is that counterconditioning to a particular disruptive stimulus generalizes to apply to other stimuli in a potentially broad class of such stimulus events. Thus, for example, Amsel, Glazer, Lakey, and McCuller (1973) obtained increased resistance to extinction by using a behaviorally disruptive tone that was presented either during or at the end of an instrumental response chain.

The two experiments reported here are investigations of the possibility that the signal-generated PREE is mediated by counterconditioning to the behaviorally disruptive effects of the signal for $\mathrm{N}$, rather than by signalgenerated retrieval and learning about $S^{\mathbf{N}}$.

There were three groups of subjects in Experiment 1. Group Signal-N was trained essentially as the Signal-N groups employed by Haggbloom (1988). In Phase 1, Group Signal-N was trained on a feature-negative discrimination problem, in which reinforced trials were not signaled and nonreinforced trials were signaled by a tactile cue that consisted of a strip of wire mesh laid on the runway floor. Phase 2 consisted of CRF training with the tactile cue occurring prior to two trials each day to generate retrieval of $S^{N}$. Group Disrupt was trained exactly as Group Signal-N in Phase 1, but in Phase 2 the subjects in Group Disrupt experienced a pretrial feeding on those trials in which Group Signal-N received the tactile cue. The pretrial feeding was novel, and thus potentially behaviorally disruptive. Group CRF received $R$ on all trials in Phases 1 and 2.

Experiment 2 was conducted exactly as Experiment 1, except that the pretrial feeding was used to signal $\mathrm{N}$ in Phase 1 in Groups Signal-N and Disrupt, and to generate retrieval of $S^{N}$ in Group Signal-N in Phase 2, and the tactile cue served as the novel stimulus for Group Disrupt. Haggbloom (1988) reported that both the pretrial feeding and the tactile cue were used successfully to produce the signal-generated PREE.

Following Phase 2 in both experiments, each group was administered three extinction trials on each of 3 days of testing.

\section{METHOD}

\section{Subjects}

The subjects in both experiments were male rats, bred in the laboratory from Holtzman stock, 90-120 days old at the beginning of training. There were 21 rats in Experiment 1,12 that were experimentally naive, and 9 that had been used previously in a serial learning experiment-in which they were all treated identically. Four naive and 3 previously used rats were randomly assigned to each of the three groups. The subjects in Experiment 2 were 9 experimentally naive rats; 3 rats were randomly assigned to each group.

\section{Apparatus}

The apparatus, fully described in Haggbloom (1988), consisted of a single straight alleyway that was $235 \mathrm{~cm}$ long, along with clocks controlled by photoelectric circuitry. The behavioral measure reported here-total speed-was measured over a $200-\mathrm{cm}$ distance from the startbox door to a point $5 \mathrm{~cm}$ in front of the goal cup. A plywood pretrial feeding box, measuring $25 \mathrm{~cm}$ on all sides and $20 \mathrm{~cm}$ high, was located on a table adjacent to the startbox. Pretrial feedings were administered in this box, from a baby food jar lid placed in a corner of the box.

\section{Procedure}

Except as noted, the same procedures were used in both experiments. The rats were housed in individual cages with access to water throughout the experiments. Two weeks prior to the start of training, the experimentally naive rats were placed on food deprivation, receiving $12 \mathrm{~g}$ of lab chow per day, minus the weight of food pellets received during training, throughout the experiment. For the previously used rats in Experiment 1 , food deprivation was reinstated after a 2-week period of free access to food. On Days 12-14 of deprivation, the naive rats were handled in squads of 2 , for 2 min per squad. After being handled on those days, the rats were fed eight $.045-\mathrm{g}$ food pellets from a small dish in their home cages.

Phase 1. Phase 1 training began on Day 15 of deprivation. Groups Signal-N and Disrupt were trained on a feature-negative discrimination problem in which all $\mathrm{R}$ trials were unsignaled and all $\mathrm{N}$ trials were signaled. The signal in Experiment 1 was a tactile cue consisting of a strip of wire mesh laid on the floor of the startbox and runway up to the goalbox door. The signal in Experiment 2 was a pretrial feeding administered by removing the rat from the intertrial holding cage and placing it in the pretrial feeding box, which was baited with eight $.045 \mathrm{~g}$ food pellets. The $\mathrm{N}$ trial was administered approximately 3-5 sec after the rat consumed the food pellets.

All subjects received six trials per day for 12 days, with three trials in each of two sessions administered at least $15 \mathrm{~min}$ apart. Within each session, the ITI was approximately $3 \mathrm{~min}$. Groups Signal-N and Disrupt received the reinforcement sequence RRN in Session 1 and RNN in Session 2 on odd-numbered days, and RNN in Session 1 followed by RRN in Session 2 on even-numbered days. Group CRF was rewarded on all trials and experienced the tactile stimulus or pretrial feedings on the same trials as Groups Signal-N and Disrupt.

Phase 2. On each of the 5 days of training in Phase 2, each subject received four $\mathbf{R}$ trials. Group Signal-N experienced the tactile stimulus (Experiment 1) or pretrial feeding (Experiment 2) as a signal prior to two trials on each CRF day. Over the 5 CRF days, the signal for $\mathbf{N}$ occurred on Trials 1 and 4,2 and 3,2 and 4, 1 and 4, and 3 and 4, respectively. Group Disrupt experienced a novel pretrial feeding (Experiment 1) or tactile stimulus (Experiment 2) on the same trials in which Group Signal-N experienced the signal for nonreinforcement. Group CRF did not experience the tactile stimulus or pretrial feedings in Phase 2.

After Phase 2, each rat received three extinction trials on each of 3 days of testing. Neither the tactile stimulus nor pretrial feedings occurred in extinction.

Trial administration procedures. Trials were begun by placing a rat in the startbox, or in the pretrial feeding box and then the startbox, depending on experimental conditions. The startbox door was raised, regardless of the rat's orientation, approximately $3 \mathrm{sec}$ after placing the rat in the startbox. In each of the three phases of training the rats were run in squads containing 1 rat from each group. The order of running rats within a squad was randomly varied across days, but the order of successive squads was held constant. The rats received eight .045-g food pellets on all $R$ trials and were confined to the goalbox for $20 \mathrm{sec}$ on all $\mathrm{N}$ trials. Pretrial feedings also consisted of eight pellets. If a rat did not enter the goalbox within $180 \mathrm{sec}$, the trial was terminated and the subject was placed in the goalbox. 


\section{RESULTS}

All analyses are based on speeds in centimeters per second $(\mathrm{cm} / \mathrm{sec})$ over the total length of the runway.

\section{Phase 1}

In Phase 1 of both experiments (data not shown), the subjects in Groups Signal-N and Disrupt learned to run slower on signaled $\mathbf{N}$ trials than on unsignaled $\mathbf{R}$ trials. In Experiment 1, for example, the difference between mean running speeds on $R$ trials as opposed to $\mathrm{N}$ trials on the last day of Phase 1 was $27.12 \mathrm{~cm} / \mathrm{sec}$ for Group Signal-N, and $42.84 \mathrm{~cm} / \mathrm{sec}$ for Group Disrupt. The comparable difference score for Group CRF was $0.06 \mathrm{~cm} / \mathrm{sec}$. In Experiment 2, the difference between mean running speeds on $\mathrm{R}$ trials as opposed to $\mathrm{N}$ trials on the 1st day of Phase 1 was $37.14 \mathrm{~cm} / \mathrm{sec}$ for Group Signal-N and $57.66 \mathrm{~cm} / \mathrm{sec}$ for Group Disrupt. The comparable difference score for Group CRF was $-7.34 \mathrm{~cm} / \mathrm{sec}$.

\section{Phase 2}

Figure 1 shows the mean difference in speed of running on signaled versus unsignaled trials for each day of Phase 2 for Experiment 1 (left panel) and Experiment 2 (right panel). The relatively large difference scores early in Phase 2 reflect the behavioral disruption caused by presentation of the signal for $\mathbf{N}$ (Signal-N groups) and by the novel stimulus (Disrupt groups). As can be seen in Figure 1, the disruptive effects of these stimuli abated over the course of Phase 2 training in both experiments.

Differences among groups at the end of Phase 2 (especially on unsignaled trials, because all extinction trials were unsignaled) could hamper interpretation of the extinction results. To evaluate behavior on unsignaled trials at the end of acquisition, a group $\times \operatorname{trial}(3 \times 2)$ ANOVA was applied to the two unsignaled trials on the last day of Phase 2 (means for these trials are shown as points A in Figure 2). The ANOVA showed there were no reliable differences among the three groups in speed of run-

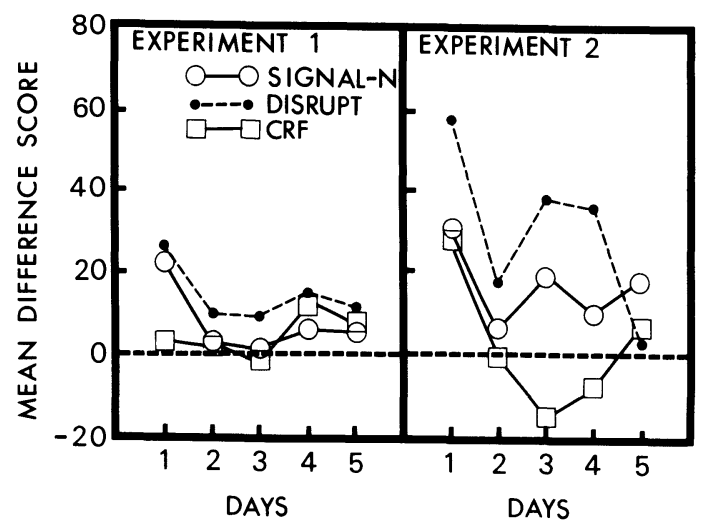

Figure 1. Mean difference between running speeds on unsignaled and signaled trials for each group on each day of Phase 2 in Experiment 1 (left panel) and in Experiment 2 (right panel).

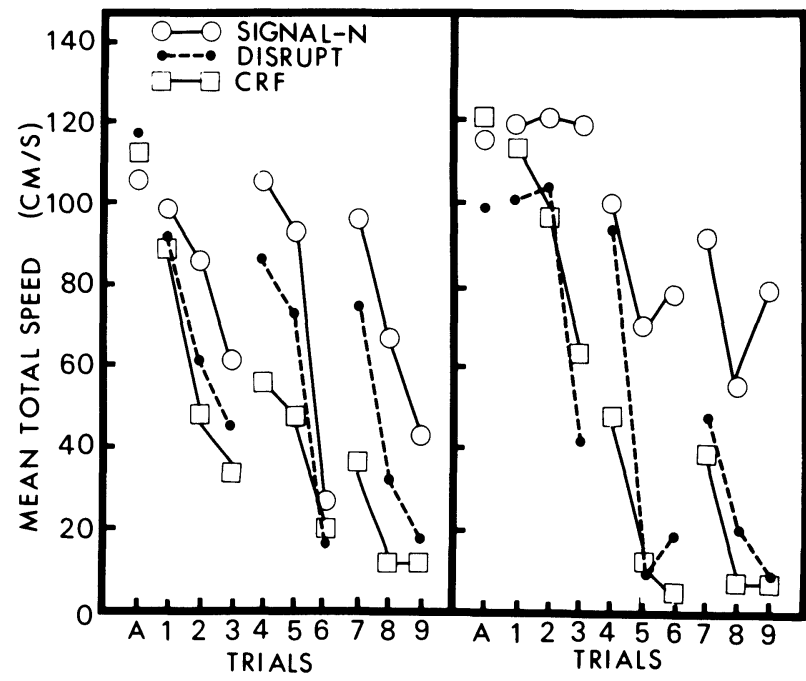

Figure 2. Mean running speed for each group on the two unsignaled trials on the last day of Phase 2 (A), and on each trial of extinction in Experiment 1 (left panel) and in Experiment 2 (right panel).

ning on unsignaled trials at the end of acquisition in either Experiment $1(F<1)$ or Experiment $2[F(2,6)=2.27]$.

\section{Extinction}

Figure 2 shows the mean speed of each group on each extinction trial in Experiment 1 (left panel) and in Experiment 2 (right panel). As can be seen, the two experiments yielded similar extinction results. In both cases, group Signal-N ran faster in extinction than Groups CRF and Disrupt. Group Disrupt ran somewhat faster than Group CRF in Experiment 1, but not in Experiment 2 . A group $\times$ trial $\times$ day $(3 \times 3 \times 3)$ ANOVA applied to the extinction data shown in the left panel of Figure 2 showed significant group differences in mean running speed over the nine extinction trials $[F(2,18)=3.61$, $p<.05]$. Planned comparisons showed that Group Signal-N ran faster in extinction than Group CRF $[F(1,18)$ $=4.92, p<.05$ ], but the mean running speed in Group Disrupt did not differ from that in Group CRF $[F(1,18)$ $=2.70]$. A comparable ANOVA applied to the data shown in the right panel of Figure 2 also yielded a reliable groups effect $[F(2,6)=16.12, p<.01]$. Planned comparisons showed that Group Signal-N ran faster than Group CRF $[F(1,6)=27.53, p<.01]$, but that Group Disrupt did not run faster than Group CRF $(F<1)$.

\section{DISCUSSION}

In both experiments reported here, as in the experiments reported in Haggbloom (1988), resistance to extinction was increased in Signal-N groups by the presentation during Phase 2 CRF training of a stimulus paired with nonreinforcement in Phase 1 . The greater resistance to extinction in Group Signal-N than in Group CRF defines the signalgenerated PREE. Haggbloom (1988) attributed the signal-generated PREE, like the conventional PREE, to conditioning of the instrumental response to $S^{N}$. In conventional PRF training, such conditioning takes place in response to event-generated $S^{N}$ stored on a nonreinforced trial 
and recalled on an immediately subsequent reinforced trial (Capaldi, 1966). Under the conditions employed here, $S^{N}$ was stored in association with the signal in Phase 1 and recalled when the signal was presented on selected R trials in Phase 2.

The purpose of the present experiments was to evaluate the possibility that the signal-generated PREE is mediated by counterconditioning of the response to a behaviorally disruptive stimulus, a possibility suggested by Amsel's generalized frustration theory (Amsel, 1972). In both experiments, responding was very substantially disrupted by the signal for $\mathrm{N}$ in the Signal-N groups and by the novel stimulus in the Disrupt groups. Over the course of Phase 2 training, there was a progressive decline in the behavioral disruption produced by these stimuli, a behavioral change that could be interpreted to reflect the underlying theoretical process of counterconditioning. However, resistance to extinction was increased only by a disruptive stimulus that signaled nonreinforcement, and not by a stimulus that was disruptive because it was novel. Thus, these experiments do not support an interpretation of the signal-generated PREE in terms of generalized frustration theory.

\section{REFERENCES}

Amsel, A. (1972). Behavioral habituation, counterconditioning, and a general theory of persistence. In A. H. Black \& W. F. Prokasy (Eds.), Classical conditioning II: Current theory and research. New York: Appleton-Century-Crofts.

amsel, A., Glazer, H., lakey, J. R., McCuller, T., \& Wong, P. T. P. (1973). Introduction of acoustic stimulation during acquisition and resistance to extinction in the normal and hippocampally damaged rat. Journal of Comparative \& Physiological Psychology, 84, 176-186.

CaPALDI, E. J. (1966). Partial reinforcement: A hypothesis of sequential effects. Psychological Review, 73, 459-477.

HAGGBLOOM, S. J. (1988). The signal-generated partial reinforcement extinction effect. Journal of Experimental Psychology: Animal Behavior Processes, 14, 83-95.

(Manuscript received July 22, 1988.) 